\title{
EXPLOTARORY ANALYSIS OF POWER SPECTRUM AND FUNCIONAL CONNECTIVITY DURING RESTING STATE IN YOUNG BINGE DRINKERS: A MEG STUDY
}

\author{
A. CORREAS \\ Laboratory of Cognitive and Computational Neuroscience, Centre of \\ Biomedical Technology (CTB), Campus Montegancedo s/n, 28223, \\ Madrid, Spain

\section{S. RODRIGUEZ-HOLGUÍN} \\ Department of Clinical Psychology and psychobiology, University \\ of Santiago de Compostela, c/ Xosé María Suárez Núñez, s/n, \\ 15982, Santiago de Compostela, Spain

\section{P. CUESTA} \\ Laboratory of Cognitive and Computational Neuroscience, Centre \\ of Biomedical Technology (CTB), Campus Montegancedo s/n, \\ 28223, Madrid, Spain

\section{E. LÓPEZ-CANEDA} \\ Department of Clinical Psychology and psychobiology, University \\ of Santiago de Compostela, c/ Xosé María Suárez Núñez, s/n, \\ 15982, Santiago de Compostela, Spain

\section{L.M. GARCÍA-MORENO} \\ Department of Psychobiology, Complutense University, c/Rector \\ Royo Villanova, s/n, 28040, Madrid, Spain

\section{F. CADAVEIRA} \\ Department of Clinical Psychology and psychobiology, University \\ of Santiago de Compostela, c/ Xosé María Suárez Núñez, s/n, \\ 15982, Santiago de Compostela, Spain \\ F. MAESTÚ \\ Corresponding author. \\ Laboratory of Cognitive and Computational Neuroscience, Centre \\ of Biomedical Technology (CTB), Campus Montegancedo s/n, \\ 28223, Madrid, Spain
}

\begin{abstract}
Binge Drinking (BD) is a pattern of intermittent intensive alcohol intake which has spread among young adults over the last decades. Adolescence constitutes a critical neuromaturation period in which the brain is particularly sensitive to the effects of alcohol. However, little is known about how BD affects to the brain activity.

This study aimed to characterize the brain's functional organization in BD and non-BD young population by means of analyzing functional connectivity (FC) and relative power spectra (PS) profiles measured with Magnetoencephalography (MEG) during eyes-closed resting state. Our sample was composed by 73 first-year university students (35 BDs and 38 controls).

Results showed that the BD subjects displayed a decreased alpha FC in frontal-parietal regions, and conversely, an enhanced FC in the delta, theta and beta bands in fronto-temporal networks. Besides the FC differences, the BD group showed a decreased PS within alpha range and an increased PS within theta range in the brain's occipital region.
\end{abstract}


These differences in FC and PS measurements provide new evidence of the neurophysiological alterations related to the alcohol neurotoxicity and could represent an initial sign of an anomalous neural activity caused by a BD pattern of alcohol consumption during youth.

Keywords: Alcohol, Binge Drinking, Resting State, Functional Connectivity, Power Spectrum, Magnetoencephalography

\section{Introduction}

Alcohol is the psychoactive substance whose use is more widespread among students aged between 14 and 18 years ${ }^{1,2}$. Over the last decades, a pattern of intermittent intensive alcohol intake, known frequently as "binge drinking", has spread among young adults ${ }^{3,4}$. Binge drinking (BD) is characterized by the intake of large amounts of alcohol in the same session, mainly during weekends, followed by a period of abstinence ${ }^{5,6}$. Although an operational definition of BD has not been used consistently, the most widely used definition for this term is the one defining it as the consumption of $60 / 40$ or more grams of alcohol (male/female) concentrated in a single session, in which the level of intoxication is maintained, at least once within the past month ${ }^{5,7}$. Definitions also refers to the duration of the drinking session, usually considering the intake of 60/40 grams of alcohol in a two-hour interval, resulting in a blood alcohol concentration of 0.08 gram percent or above $^{7,8}$.

For many years, most of the research on alcohol brain damage has focused on chronic alcoholism. More recently, the interest of neurotoxicity induced by $\mathrm{BD}$ during adolescence has arisen. Adolescence is a critical neuromaturation period during which the brain is particularly sensitive to the effects of alcohol ${ }^{9,10}$. The region that experiences the most notable changes is the prefrontal cortex (PFC), which does not reach maturity until early adulthood ${ }^{11}$. The maturation of the PFC supports the improvement of complex behavioral functions and cognitive skills, such as working memory, planning, problem solving or inhibitory control ${ }^{12}$.

Recent studies reveal that alcohol use disorders (AUD) in human adolescents may cause structural damage, especially in the PFC and the hippocampus ${ }^{13-15}$. Cognitive deficits consistent with damage in the PFC have also been reported in BD population, both with and without AUD, especially in working memory, decision making, problem solving, flexibility and inhibitory control ${ }^{16-23}$. Furthermore, two studies have found white matter integrity damage when measure DTI associated to the DB consumption ${ }^{24,25}$. EEG studies assessing event-related potentials (ERP) have provided evidence about differences between $\mathrm{BD}$ and Control groups during performance of different tasks involving working memory, inhibition response or processing emotional stimuli $^{26-32}$.

Considering the need of a more comprehensive research within this issue, the aim of this study was to explore the potential neurophysiological alterations during resting state associated with a BD pattern of alcohol consumption. Resting state comprises the activity of selective brain regions when the brain is not involved in an externally imposed goal-directed activity, and constitutes a core feature of brain activity. This paradigm is broadly studied nowadays probably due to the fact that its degeneration has been associated with impairments in different cognitive processes ${ }^{33-35}$ as well as with several neuropsychiatry disorders ${ }^{36-39}$, including alcoholism ${ }^{40-42}$. Thus, alterations in the brain resting state activity could be used to give an indication of potential neurophysiological impairments related to BD.

In the present study, eyes-closed resting state was recorded with Magnetoencephalography (MEG). The MEG is a noninvasive technique which measures directly the magnetic fields of the brain activity with millisecond accuracy. This system offers a different approach to fMRI, which measures directly the hemodynamic response, and from this response it infers the brain activity. In addition, the MEG has been proven to be useful when studying both spontaneous and taskrelated brain activities $35,38,43$. As a first step, we calculated the Power Spectrum (PS), which may reflect the organized activity in local networks of neurons, but per se it does not capture the dynamical interactions among the distributed neural assemblies. There is increasing evidence showing that the communication among these neural networks takes place through the synchronization of their activity. This Synchronization can be assessed by means of Functional Connectivity (FC) ${ }^{44,45}$, which measures statistical interdependences among the physiological signals which emerge from different brain areas. Among the numerous algorithms which measure the $\mathrm{FC}^{46}$, the most used in neuroscience are those that assess the phase of the physiological signals. One of them is the Phase Locking Value (PLV) 47 or mean phase coherence, which offers a straightforward estimation of the averaged phase coupling in each frequency band, among different electrophysiological brain signals. Therefore, under the FC approach, the brain is a complex neural network, 
where the FC represents the communication that takes place among different regions. At the same time, there is evidence about the correlation between FC values and cognitive functions such as language, attention or memory ${ }^{48-50}$. Thus, the disturbance of synaptic transmission, the loss of neurons or the degeneration in the integrity of the white matter tracts are some of the biological processes that may affect the ability to integrate information from different brain regions and therefore, may impair the FC.

FC has showed to be impaired in subjects with chronic alcoholism. FMRI studies have revealed that FC networks are affected among these patients during different task performance ${ }^{51-54}$, and resting state. Chanraud and cols. found a reduction of synchrony between posterior cingulate and cerebellar regions in alcoholics compared to controls during resting state. It must be taken into account that the fMRI studies calculate FC from the brain hemodynamic response; on the other hand, MEG FC is calculated from the cerebral magnetic activity. Therefore, since they are signals of different nature, the relationship between them must be made cautiously. As far as we know, only one study measured with EEG has assessed FC in young students with high and regular alcohol consumption during resting state ${ }^{55}$. Bruin and cols. compared heavy alcohol drinkers with moderate and light drinkers and they found that those students who drank more had higher theta and gamma Synchronization Likelihood (SL).

To the best of our knowledge, this is the first neurophysiological investigation that assesses the PS profile and FC pattern during brain resting state in BD subjects as compared to light-drinker controls. We tested the hypothesis that the BD pattern of alcohol consumption may induce anomalies in the oscillatory and synchronized brain activity, even in early ages.

\section{Methods and Materials}

\subsection{Subjects}

MEG signals were obtained from 73 first-year students of the University Complutense of Madrid: 35 BDs (17 males and 18 females) and 38 control subjects ( 21 males and 17 females). They were divided into binge drinkers (BD group) and a control group according to a questionnaire and a semi-structured interview inquiring about alcohol and other drug consumption habits. Participants were asked to cover a record of daily consumption indicating what they drank, the quantity and for how long (hours). Their blood alcohol concentration (BAC) was calculated based on the information of drinking episode of the last 6 months and according to the following algorithm:

$B A C=\left(\frac{\text { G of alcohol consumed }}{\text { Weight in } \mathrm{Kg} \times r}\right)-m r \times$ hours

where $r$ is a constant with value 0.68 for male and 0.55 for female and $m r$ the metabolization rate with value 0.15 for male and 0.18 for female. We considered the $\mathrm{BAC}$ value as a rough index representing the $\mathrm{BD}$ level of each subject. Participants reaching BAC of $0.08 \%$ or above, which is a binge drinking episode, at least once during the last month were classified as BD. On the other hand, the control group was consisted of students who never achieved that alcohol concentration. The average time that the $\mathrm{BD}$ group has been drinking is $14.98 \pm 1.17$, making it a very homogeneous history of consumption, so this question would not be a contaminating variable. Demographic data are shown in Table 1.

Table 1. Demographic, Tobacco, Drinking and number of trials data

\begin{tabular}{lcc}
\hline & Control & BD \\
\hline & & \\
N (males/females) & $38(21 / 17)$ & $35(17 / 18)$ \\
Age & 18 & 18 \\
Tobacco smokers & 0 & 4 \\
Grams of alcohol in a BD & $0.0147 \pm$ & $0.1537 \pm$ \\
day (BAC)* & 0.024 & 0.0413 \\
Number of trials & $39.1 \pm 7.4$ & $37.6 \pm 8.9$ \\
\hline
\end{tabular}

The alcohol grams present in a "Standard Unit Drink" (SUD) varies from country to country and therefore, these differences make it necessary to adapt the criteria about number of SUDs to the country in which the study is being carried out. As it was mentioned before, the consumption of $60 / 40$ or more grams of alcohol concentrated in a single session is considered a binge drinking episode, and, in Spain, that amount corresponds to 5/4 SUD or above ${ }^{5}$, which produces a BAC of $0.08 \%$. 
All volunteers provided written informed consent prior to assessment. Participants were asked to refrain from alcohol consumption for, at least, 24 hours prior to MEG recordings. Subjects were submitted to a breathalyzer test, and the assessment was only performed after verifying a $0.00 \%$ breath alcohol level. The exclusionary criteria of the study are shown in Table 2. In order to minimize the possible influence of a genetic predisposition for alcoholism, participants were questioned about their personal and family history of alcoholism (FHA). Personal history of psychopathological disorders (axes 1 and 2) were excluded according to DSM-IV-TR criteria and psychopathological traits were assessed by the Symptom Cheklist-90 Revised questionnaire (SCL-90-
R). In addition, it was verified that subjects neither have any disease affecting cognitive functioning or neurological disorders nor were taking any medicine with psychoactive effects. Besides, they answered the Alcohol Use Disorders Identification Test (AUDIT) and subjects who scored 20 or above were excluded for having alcohol use disorders. Tobacco consumption was not an exclusion criterion but a controlled variable. According to the Syndrome Nicotine Dependence Scale (NDSS-S) ${ }^{56}, 4$ participants fulfilled the criterion of nicotine dependence, all belonging to the $\mathrm{BD}$ group. Regarding cannabis consumption, although only regular users of cannabis were excluded of the sample, no subject consumed even occasionally.

Table 2. Exclusion criteria applied in this study.

Medical conditions affecting the normal cognitive functioning
Personal history of neurological disorder
Personal history of psychopathological disorders (according to DSM-IV criteria)
Family history of major psychopathological disorders in first degree relatives
Family history of first or second degree of alcoholism or substance abuse
Use of illegal drugs (except occasional cannabis consumption)
Megular consumption of medical drugs with psychoactive effect (sedatives/anxiolytics)
Motor sensory disabilities uncorrected
AUDIT scores $\geq 20$

\subsection{MEG recordings}

Four minutes of resting state with eyes closed were acquired at $1000 \mathrm{~Hz}$ sampling rate (online bandpass filtering at $0.1-330 \mathrm{~Hz}$ ) with a 306-channel Vectorview system (ElektaNeuromag) which combines two orthogonal, planar gradiometers, and one magnetometer. Only magnetometers (102 channels) information was analyzed in this study. The system was housed in a magnetically shielded room (VacuumSchmelze $\mathrm{GmbH}$, Hanua, Germany) at the "Laboratorio UPM-UCM de Neurociencia Cognitiva y Computacional" (Madrid, Spain). The head movement was controlled by means of four head-position indicator (HPI) coils attached to the scalp. The position of HPI coils and subject's headshape placed on three anatomical locations (nasion and both preauricular points) was defined using a 3D digitizer (FastrakPolhemus). Ocular movements were tracked by means of two bipolar electrodes. Recordings were submitted to Maxfilter software (v 2.2, correlation threshold $=0.9$, time window $=10$ seconds) in order to remove external noise with the temporal extension of the signal space separation method with movement compensation ${ }^{57}$. Resting state magnetometer's data was automatically scanned for ocular, muscle and jump artifacts with Fieldtrip package ${ }^{58}$. Artefact-free data were segmented into continuous 4-second fragments (trials). The MEG PS $(1-45 \mathrm{~Hz})$ was computed for all trials (see below for details). An experienced technician, blinded to the subjects' group, carried out a visual inspection over the raw data and the spectra. Those trials with noisy raw signal or aberrant PS profile were dismissed. Finally, only MEG recordings with at least 15 clean trials (one minute of brain activity) were kept for further analyses. The number of surviving trials did not differ significantly between groups (control group: $(39.1 \pm 7.4)$, binge group: $(37.6 \pm 8.9), \mathrm{p}=0.75)$. Matlab version 8.0 (Mathworks, Natick, MA, USA) was used for the analysis with custom-written scripts.

\subsection{Power spectrum}


MEG PS were computed through Fieldtrip with a variable frequency of interest range of $0.5 \mathrm{~Hz}$ steps from 2 to $25 \mathrm{~Hz}$ and $1 \mathrm{~Hz}$ steps from 26 to $45 \mathrm{~Hz}$. The average frequency-content of each trial was obtained through a multitaper method (mtmfft) with discrete prolate spheroidal sequences (dpss) as windowing function and $1 \mathrm{~Hz}$ smoothing. These PS were averaged across trials, obtaining for each subject a matrix whose dimensions were 102 channels x 67 frequency steps. Finally, the PS was normalized with the sum of the spectral power in the $2-45 \mathrm{~Hz}$ range ${ }^{59,60}$.

\subsection{Phase locking value}

In this study, the FC was measured by phase-locking value (PLV) ${ }^{47}$ in the following frequency bands: delta (2-3.9 Hz), theta $(4.1-7.9 \mathrm{~Hz})$, alpha $(8.1-11.9 \mathrm{~Hz})$ and beta $(12.1-29.9 \mathrm{~Hz})$. First, the time series were filtered with a Finite Impulse Response filter of order 300 designed with a Hamming window. The filter was applied using a two-pass procedure over the whole fourminute registers, in order to avoid phase distortion and edge effects. The starting data set consisted in matrices with dimensions: 102 channels x 4000 samples x 4 frequency bands $x$ trials. Then, for each frequency band and trial, we have calculated PLV via the following procedure: (1) for each sensor $\mathrm{j}=1 \ldots 102$, the phase of the signal $x_{j}(t)$ was extracted by means of a Hilbert transform:

$$
z_{j}(t)=x_{j}(t)+i \cdot \operatorname{Hilbert}\left(x_{j}(t)\right)=A_{j}(t) \cdot e^{i \varphi_{j}(t)}
$$

(2) the synchronization between a pair of phases $\varphi_{j}(t)$ and $\varphi_{k}(t)$ was calculated with the following expression:

$$
P L V=\frac{1}{M}\left|\sum_{m=1}^{M} e^{i\left(\varphi_{j}\left(t_{m}\right)-\varphi_{k}\left(t_{m}\right)\right)}\right|
$$

Where $\mathrm{M}=4000$ is the number of samples in the time series (4 seconds sampled at $1000 \mathrm{~Hz}$ ). Finally, the results were averaged across trials ending up with symmetrical 102 channels x 102 channels connectivity matrices for subject and frequency band.

\subsection{Statistical analysis}

In both cases (PS and FC analysis), the first step consisted in transform, prior to the statistical tests, the values (power or PLV) by means of

$x=\log (x / 1-x)$

in order to obtain values following a normal distribution 60

\subsubsection{Power Spectrum Analysis}

In order to accomplished a data-driven analysis of the power differences between groups we use a methodology designed for EEG/MEG data extracted from the cluster based non parametric permutation test described by Maris and Oostenveld ${ }^{61}$. A similar methodology has been employed successfully in previous studies ${ }^{59,62,63}$.

First, an exploratory pairwise t-test was calculated per each channel in each frequency step. Those comparisons which were found to show significant differences between groups $(\mathrm{p}<0.05)$ were clustered according to a criterion of spatial (each cluster must contain at least 3 contiguous and significant sensors) and frequency adjacency (the difference between pairs of groups must remain significant during at least a $2 \mathrm{~Hz}$-interval which corresponds to 4 frequency steps). Then, the obtained power values were submitted to a non-parametric permutation test. This test consisted in assign randomly 2000 times the power values to the original groups. The sum of $t$-values over each cluster in the original data set was compared with the same measure in the randomized data. Therefore, for each cluster, the proportion of randomizations with $\mathrm{t}$-values higher than the ones in the original data corresponds to the final p-value. Finally, with the aim of characterizing the significant differences between groups in each cluster and frequency range; we carried out another t-test between the averaged power values in the significant clusters and within the significant frequency ranges. This comparison was submitted as well to the non-parametric test, which was explained before. These are the values shown in the results section.

\subsubsection{Functional Connectivity Analysis}

The FC statistical analysis was based as well in the methodology introduced by Maris and Oostenveld. The procedure was carried out independently for each 
frequency band. First, an exploratory t-test was calculated per each PLV value. Then, those channels with at least two significant links were taken into account as members the significant network. These PLV values were submitted to the non-parametric test explained before. The last step and similarly to the power analysis procedure, we calculated another t-test between groups with the averaged PLV value across each significant network. This comparison was checked via the non-parametric test and corresponds with the values shown in the results section.

In addition, Spearman correlation test in the BD sample were calculated between the significant average values (both, PS and FC) and the BAC score. These scores were checked as well by means of corresponding nonparametric test.

Finally, the effect sizes of the significant results were calculated through the following expression:

$\Delta=\frac{\overline{X_{B D}}-\overline{X_{C N}}}{\sigma_{C N}}$

where $\sigma_{C N}$ was the standard deviation of the control group. The $\overline{X_{B D}}$ and $\overline{X_{C N}}$ values corresponded with the average significant value (PS or FC) of the BD and control group respectively.

\section{Results}

\subsection{Relative Power Spectra}

The average relative power in the $1-45 \mathrm{~Hz}$ range was obtained for each group. We found differences only within the first third of the spectra, so only this section is depicted in figure 1 . The profile of the spectral distribution was different for both groups. Both showed the maximum peak around $10 \mathrm{~Hz}$ frequency and significant differences between groups were found within two ranges. (1) A range within theta band that included frequencies between 4-6 Hz (henceforth called theta range), with $\mathrm{BD}$ group displaying increased theta range power in an occipital cluster of sensors compared to the control group $(\mathrm{t}=-2.014, \mathrm{p}=0.044)$. (2) A range within alpha band that included frequencies between 9 $11 \mathrm{~Hz}$ (henceforth called alpha range), where the BD group showed reduced alpha range power in a temporaloccipital cluster of sensors as compared to the control group $(t=-2.294, p=0.025)$. The effect size values per the comparison of the average significant power of theta and alpha ranges are 0.64 and -0.51 respectively.

The possible gender influence was tested for all results through an extra two way ANOVA test with gender and group as main variables. Neither gender main effect nor gender by group interaction were found significant $(\mathrm{p}>$ $0.05)$. 

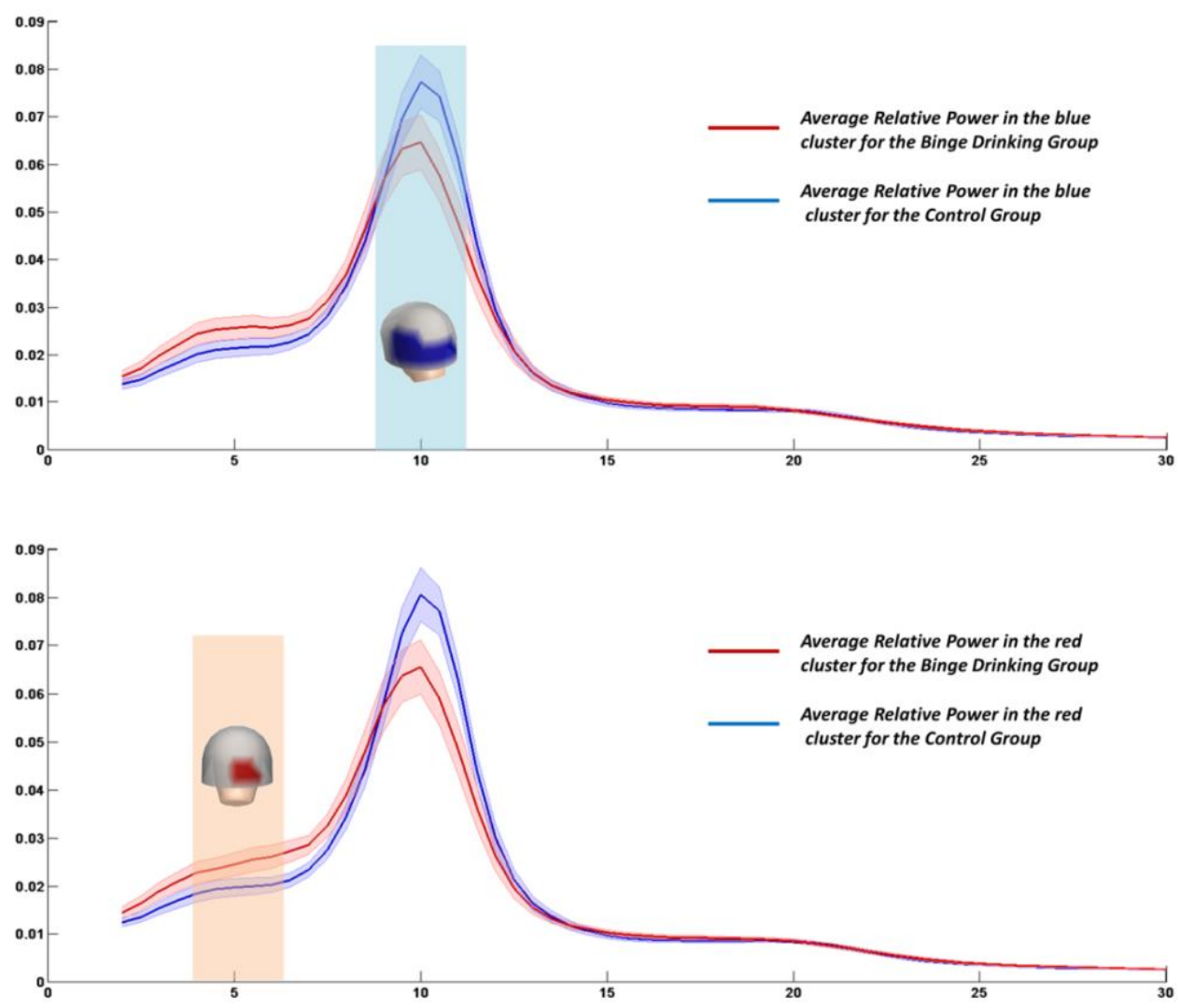

Fig. 1. Power spectra results. Top, differences within the alpha band range $[9-11 \mathrm{~Hz}]$. The cluster in blue color represents a decrease in relative power in the binge drinking group in temporal-occipital regions in comparison with the control group. Red and blue lines depicted the average relative power spectra for the channels of the blue cluster in the binge drinking group and control group, respectively. Highlighted region in blue refers to the frequency range with significant differences in the blue cluster of channels. Bottom, differences within the theta band range $[4-6 \mathrm{~Hz}]$. The cluster in red color represents an increase in relative power in the binge drinking group in the occipital region in comparison with the control group. Red and blue lines depicted the average relative power spectra for the channels of the red cluster in the binge drinking group and control group, respectively. Highlighted region in orange refers to the frequency range with significant differences in the red cluster of channels. Spectra are represented on the " $\mathrm{x}$ " axis from 2 to $30 \mathrm{~Hz}$ frequency whilst the " $y$ " axis depicts relative power values. Shaded error bars display corresponding standard error. The effects size values per the comparison of the average significant relative power of theta and alpha ranges are 0.64 and -0.51 respectively.

\subsection{Functional Connectivity}

Regarding FC, significant differences were found in delta, theta, alpha and beta bands. In delta, theta and beta the FC of the BD group was enhanced compared with the FC of the control group, whilst in alpha band the BD group showed diminished FC.

In delta band, a hyper-synchronized network in the BD group was located by connecting the right frontal and right temporal areas $(t=-3.3883, \mathrm{p}=0.0012)$. When the FC in theta band was analyzed, a network connecting the middle frontal and the middle parietal areas showed increased FC in the BD group relative to the control group $(\mathrm{t}=-2.8471, \mathrm{p}=0.0058)$. Another network with enhanced FC was found in beta band, where an increased long range $\mathrm{FC}$ was found in the BD group between right frontal and right temporal regions $(\mathrm{t}=$ $3.6786, \mathrm{p}=0.0005)$. Finally, the FC analysis in alpha band showed diminished synchronization in the BD group in a long range network, which connected the left frontal and left temporal areas $(\mathrm{t}=2.8585, \mathrm{p}=0.0056)$ (see Figure 2). Significant values of FC in alpha band 
correlated negatively with the $\mathrm{BAC}$ value of the $\mathrm{BD}$ group (rho $=-0.56, p=0.00088$ ), therefore, the more $\mathrm{BAC}$, the less alpha FC between the left frontal and the left temporal areas.

The effect size values per the comparison of the average significant FC delta, theta, alpha and beta bands are $0.64,6.69,-0.65$ and 0.87 respectively.
The possible gender influence was tested for all results through an extra two way ANOVA test with gender and group as main variables. Neither gender main effect nor gender by group interaction were found significant $(\mathrm{p}>$ $0.05)$.

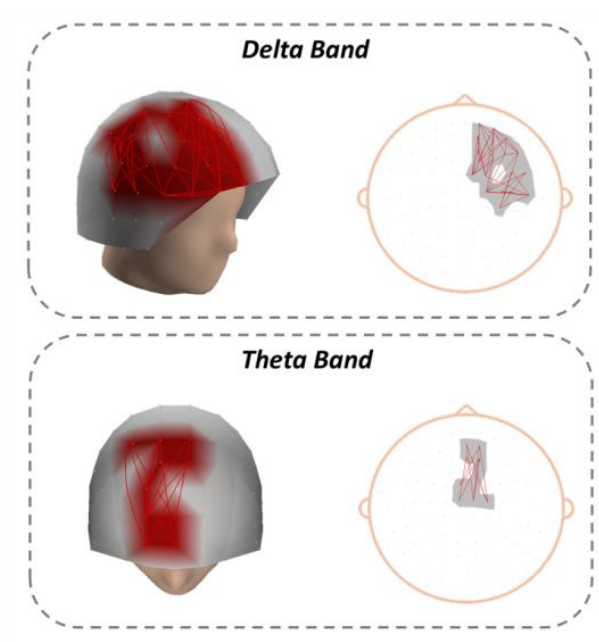

FC Binge Drinking Group > FC Control Group

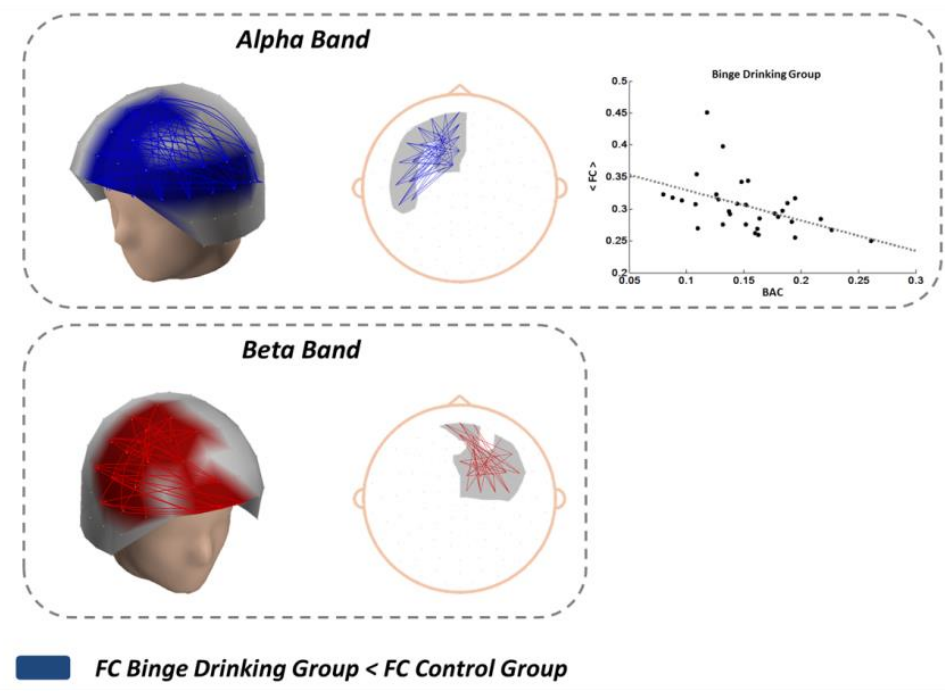

Fig. 2. Functional connectivity results. This figure shows the significant network for each frequency band. 3D and 2D models show the same networks. The FC of the control group is employed as the reference. Therefore, a line between two channels represents a link whose FC value has been found significantly different between both groups. The red/blue color indicates that the FC of the binge drinking group has been found enhanced/diminished in comparison to the FC of the control group. The effects sizes values per the comparison of the average FC of the delta, theta, alpha and beta significant networks are $0.64,0.69,-0.65$ and 0.87 respectively. For alpha band, the scatter plot depicts the significant Spearman correlation between the average FC of the alpha significant network and the BAC score in the binge drinking population $(\mathrm{p}=0.0002$, rho $=-0.56)$.

\section{Discussion}

The results of the present study showed that FC and PS, as assessed by MEG signal during eyes-closed resting state, differ between young control and BD students. The statistical analysis indicated that: 1) the BD group, compared to the control group, depicted diminished PS in alpha and enhanced in theta frequency ranges in occipital areas; 2) FC analysis showed a significant increase in the BD group in delta, and beta bands in right fronto-temporal networks, and also in the theta band in a middle fronto-parietal network, together with a significant decrease in alpha synchronicity between left frontal and parietal regions.

\subsection{PS differences}

Given that several studies evaluating PS during resting state in alcoholics have also found an increase in theta ${ }^{64}$ as well as a decrease in alpha ${ }^{65,66}$ bands, our results seem to indicate that the BDs might exhibit a similar spectral pattern as alcoholics in these frequency bands. Regarding the theta band, it is known that this frequency band decreases across individual development ${ }^{67,68}$. In the same way, reductions in PS of theta bands have been related to the reduction that naturally occurs in the cerebral gray matter volume from childhood to young adulthood ${ }^{69}$. Inasmuch as some studies have associated the BD pattern of alcohol consumption in youths with a potential neuromaturational delay, i.e., with greater grey matter volumes in certain cortical and subcortical regions due to a lower synaptic pruning ${ }^{70-72}$, the greater theta band observed in BDs compared with controls could be related with a neurodevelopmental delay in these subjects. However, this interpretation is still tentative 
and new studies assessing PS and cortical thickness are necessary to test this hypothesis.

Regarding alpha band, there is considerable evidence that a resting state eyes-closed condition in healthy subjects induces an oscillatory activity around $10 \mathrm{~Hz}$ over the posterior scalp regions ${ }^{73}$. Large alpha power in a resting state period has been related to good memory performance during a memory task ${ }^{49,74}$. Thus, the decrease in alpha band in occipital areas of the BD group may be related to a deficit in memory compatible with cognitive studies with this population showing deficits in memory tasks ${ }^{21,22,75}$.

Besides, a recent study has shown that acute alcohol intoxication also affects alpha band, increasing its short-term power mainly during eye-closed resting state, also measured by MEG ${ }^{76}$. Therefore, it would be possible that a pattern of consumption that includes repeated alcohol intoxication, as BD, affects long-term PS and, specifically to alpha power.

To our knowledge, only one study has evaluated PS in BDs. In this study, Courtney and Polich (2010) found differences in PS measured by EEG signal between university students who varied in their alcohol consumption ${ }^{77}$. High-binge drinkers (> 10 drinks in under two hours on more than one occasion within the past six months) exhibited increased PS in the delta and fast-beta $(20-35 \mathrm{~Hz})$ bands as compared to nonand low-binge drinkers (5/4-7/6 drinks under two hours). However, there were no differences between non-binge drinkers and low-binge drinkers in these frequency bands. In our study, controls (light drinkers) and BD subjects display similar patterns of alcohol consumption as the non- and low-BDs observed in the Courtney and Polich's study. It must be noted that our $\mathrm{BD}$ sample is equivalent to the low-BD group in that study. Thus, the absence of differences in the delta and fast-beta frequency ranges of our study is consistent with the results presented by these authors. On the other hand, we do find PS differences between our groups, unlike the absence of differences between nonand low-binge drinkers in their study. The divergence between both results might be due to the differences between the two studies: EEG vs. MEG recording, eyes-open (EO) vs. eyes closed (EC) resting state, or the number of subjects in the sample. PS significant differences found in this study were mainly located in occipital MEG sensors, typical areas where alpha is activated during resting state. Differently, Courtney and Polich's EEG differences were found in the $\mathrm{Cz}$ electrode. Another difference between studies is that PS of resting state EC varies form EO and also its spatial distribution. The major difference between conditions is the reduction of alpha band from EC resting state to EO ${ }^{78}$. New EEG and MEG recordings will be necessary to clarify the alteration patterns during resting state in young people with a BD pattern of alcohol consumption.

\subsection{FC differences}

As stated in the introduction, the loss of white matter integrity associated may entail a lower efficiency in the communications among brain regions and therefore, we think that it may affect the FC and the PS. In this sense, several studies using fMRI have observed disturbances in $\mathrm{FC}$ in alcoholic patients during resting state ${ }^{40}$ as well as during performance of different cognitive tasks ${ }^{51-54}$. Another study conducted by Bruin and cols. (2006) showed impaired synchronization of the brain activity (reflected as lower alpha and slowbeta activity in the EEG) during resting state in a population of adults heavy drinkers, whose alcohol consumption was between 21 and 53 drinks per week 79 . However, in this study the main result involved a heavy and regular drinker population, which does not match with our sample of BDs.

While these studies in subjects with alcohol dependence or abuse report impairments in neural networks, it is still questioned whether BD could cause connectivity disruption. In this sense, as stated in the introduction, two studies from the same laboratory observed that white matter integrity was also compromised in young BDs in several association and projection white matter tracts, such as -occipital and fronto-temporal connections ${ }^{24,25}$. Altered white matter integrity could partially underlie functional alterations in $\mathrm{BD}$ population observed in these regions. To our knowledge, no study has evaluated the FC networks in this population during resting state. Only one study examined the brain FC in young subjects (aged 22-27 years) with a pattern of consumption of 30 or more alcoholic drinks per week during resting eyes closed state. This regular and heavy alcohol consumption, although different from de BD pattern, seems to induce FC disruptions partially similar to those observed in the BD group of our study. In that study, the heavy alcohol drinkers displayed higher synchronization in the theta and gamma bands as compared to the control group. The augmented theta synchronization in these heavily drinking students resembles the increased theta FC in the BDs students of our study.

Our results also showed a diminished synchronization between left frontal and left temporal areas in the BD group. As stated above, alpha rhythm is the most dominant in resting state, strongly related with several cognition processes $49,50,80$, and it also has an important functional role in the inhibitory processes ${ }^{81-83}$. There is growing evidence about the importance of the frontoparietal regions in efficient cognitive control ${ }^{84,85}$. But more important seems to be the interaction of alpha rhythm with those brain regions which are suppressed during cognitive control tasks ${ }^{86}$. This interaction, consisting in a switch between externally and internally oriented cognition, is really important for cognitive 
performance ${ }^{87}$, even in resting state ${ }^{88}$. So the inhibition role of alpha band could be an effective mechanism to regulate the output of cortical networks ${ }^{89}$. Several studies have found that BD adolescents perform poorly in task involving prefrontal activity ${ }^{16,18}$, and especially in inhibitory control processes ${ }^{19,20}$. In this sense, the diminished FC of the BD group in alpha band involving left frontal and temporal areas at rest found at this study could represent a sign of neural anomaly accountable for the poor performance in inhibitory processes in this population. This lack of inhibition might increase impulsivity contributing to the development, persistence and/or severity of alcohol use disorders ${ }^{90,91}$.

Furthermore, another proof that reinforces the fact that the BD pattern produces a FC decrease in alpha band is the negative correlation between this decline and the increase of the BAC score, therefore, the more BAC, the less alpha FC between the left frontal and the left temporal areas.

Regarding the increased FC in delta, theta and beta bands, enhanced FC has also been observed in several abusing groups of substances, such as alcohol, opioids or cannabis ${ }^{63,92-95}$. This increased FC has often been interpreted as a compensatory mechanism for the reduced or altered FC in other regions. Other studies with adolescent and young students have concluded that BD pattern can lead to a neural over-activation, in spite of the absence of behavioral performance differences ${ }^{29,30,96,97}$. This neural hyperactivity has also been explained as compensatory cerebral changes in order to facilitate normal behavior performance. Thus, it is possible to think that the increased FC in delta, theta and beta bands might result from a compensation of the decrease of alpha band, but we are not able to validate it without further neuropsychological information.

A common limitation that affects studies with this type of population is that the cross-sectional nature of this study makes it difficult to draw a conclusion about casual relationships between the neurophysiological differences and the binge drinking consumption. It cannot be excluded that the differences between groups are previous to the consumption. In spite of this limitation, we were still able to demonstrate reliable neurophysiological differences between a BD group and control subjects.

\subsection{Conclusions}

To the best of our knowledge, this is the first study assessing the FC patterns together with the analysis of PS profiles measured by MEG in young subjects with a BD pattern. Differences found in this study between BDs and light or non-drinkers in PS and FC measurements could represent an initial sign of an abnormal oscillatory and synchronized neural activity associated to a BD pattern of alcohol consumption during youth. The high prevalence of this behavior among the young population increases the necessity of further studies in order to confirm the results and to clarify the dimension of this problem.

\section{Reference}

1. Eurobarometer, EU citizens' attitudes towards alcohol. (2010)

2. Johnston, L., Monitoring the Future: National Results on Adolescent Drug Use: Overview of Key Findings (Google eBook). (2010)

3. O. E. de las D. y las T., Informe Nacional 2012 (datos 2011) al OEDT por el Punto Focal Nacional (ESPAÑA). (2012)

4. Substance Abuse and Mental Health Services Administration, Results from the 2012 National Survey on Drug Use and Health: Summary of National Findings, NSDUH. (2013)

5. Parada, M. et al., Definition of adolescent binge drinking. Adicciones 23 (2011) 53-63

6. Wechsler, H., Davenport, A., Dowdall, G., Moeykens, B. \& Castillo, S., Health and behavioral consequences of binge drinking in college. A national survey of students at 140 campuses. JAMA 272 (1994) 1672-7

7. Courtney, K. E. \& Polich, J., Binge Drinking in Young Adults: Data, Definitions and Determinants. Psychol bull 135 (2009) 142-156

8. Department of Health and Human Services. National Institutes of Health, NIAAA Newsletter. Number 3. (2004)

9. $\quad$ Crews, F., He, J. \& Hodge, C., Adolescent cortical development: a critical period of vulnerability for addiction. Pharmacol. Biochem. Behav. 86 (2007) 189-99

10. Barron, S. et al., Adolescent vulnerabilities to chronic alcohol or nicotine exposure: findings from rodent models. Alcohol. Clin. Exp. Res. 29 (2005) $1720-5$

11. Lenroot, R. K. \& Giedd, J. N., Brain development in children and adolescents: insights from anatomical magnetic resonance imaging. Neurosci. Biobehav. Rev. 30 (2006) 718-29

12. Spear, L. P., The adolescent brain and age-related behavioral manifestations. Neuroscience and biobehavioral reviews 24 (2000)

13. De Bellis, M. D. et al., Prefrontal cortex, thalamus, and cerebellar volumes in adolescents and young adults with adolescent-onset alcohol use disorders and comorbid mental disorders. Alcohol. Clin. Exp. Res. 29 (2005) 1590-600

14. Medina, K. L. et al., Prefrontal cortex volumes in adolescents with alcohol use disorders: unique gender effects. Alcohol. Clin. Exp. Res. 32 (2008) 386-94

15. De Bellis, M. D. et al., Hippocampal volume in adolescent-onset alcohol use disorders. Am. $J$. Psychiatry 157 (2000) 737-44

16. Scaife, J. C. \& Duka, T., Behavioural measures of frontal lobe function in a population of young social drinkers with binge drinking pattern. Pharmacol. Biochem. Behav. 93 (2009) 354-62

17. Hartley, D. E., Elsabagh, S. \& File, S. E., Binge drinking and sex: effects on mood and cognitive function in healthy young volunteers. Pharmacol. Biochem. Behav. 78 (2004) 611-9

18. García-Moreno, L. M., Expósito, J., Sanhueza, C. \& Angulo, M. T., [Prefrontal activity and weekend 
alcoholism in the young]. Adicciones 20 (2008) 271-9

19. Townshend, J. M. \& Duka, T., Binge Drinking, Cognitive Performance and Mood in a Population of Young Social Drinkers. Alcohol. Clin. Exp. Res. 29 (2005) 317-325

20. Nederkoorn, C., Baltus, M., Guerrieri, R. \& Wiers, R. W., Heavy drinking is associated with deficient response inhibition in women but not in men. Pharmacol. Biochem. Behav. 93 (2009) 331-6

21. Mota, N. et al., Binge drinking trajectory and neuropsychological functioning among university students: a longitudinal study. Drug Alcohol Depend. 133 (2013) 108-14

22. Parada, M. et al., Binge drinking and declarative memory in university students. Alcohol. Clin. Exp. Res. 35 (2011) 1475-84

23. Parada, M. et al., Executive functioning and alcohol binge drinking in university students. Addict. Behav. 37 (2012) 167-72

24. Jacobus, J. et al., White matter integrity in adolescents with histories of marijuana use and binge drinking. Neurotoxicol. Teratol. 31 (2009) 349-55

25. McQueeny, T. et al., Altered white matter integrity in adolescent binge drinkers. Alcohol. Clin. Exp. Res. 33 (2009) 1278-85

26. Ehlers, C. L. et al., P3 components and adolescent binge drinking in Southwest California Indians. Neurotoxicol. Teratol. 29 (2007) 153-63

27. Petit, G. et al., Early attentional modulation by alcohol-related cues in young binge drinkers: an event-related potentials study. Clin. Neurophysiol. 123 (2012) 925-36

28. Crego, A. et al., Increased amplitude of P3 eventrelated potential in young binge drinkers. Alcohol $\mathbf{4 6}$ (2012) 415-25

29. López-Caneda, E. et al., Hyperactivation of right inferior frontal cortex in young binge drinkers during response inhibition: a follow-up study. Addiction 107 (2012) 1796-808

30. López-Caneda, E. et al., Effects of a persistent binge drinking pattern of alcohol consumption in young people: a follow-up study using event-related potentials. Alcohol Alcohol 48 464-71

31. Crego, A. et al., Binge drinking affects attentional and visual working memory processing in young university students. Alcohol. Clin. Exp. Res. 33 (2009) 1870-9

32. Crego, A. et al., Reduced anterior prefrontal cortex activation in young binge drinkers during a visual working memory task. Drug Alcohol Depend. 109 (2010) 45-56

33. Esposito, F. et al., Does the default-mode functional connectivity of the brain correlate with workingmemory performances?. Arch. Ital. Biol. 147 (2009) $11-20$

34. Sambataro, F. et al., Age-related alterations in default mode network: impact on working memory performance. Neurobiol. Aging 31 (2010) 839-52

35. Ahmadlou, M., Adeli, A., Bajo, R. \& Adeli, H., Complexity of functional connectivity networks in mild cognitive impairment subjects during a working memory task. Clin. Neurophysiol. 125 (2014) 694-702

36. Damoiseaux, J. S. et al., Reduced resting-state brain activity in the "default network" in normal aging. Cereb. Cortex 18 (2008) 1856-64

37. Kennedy, D. P. \& Courchesne, E., Functional abnormalities of the default network during self- and other-reflection in autism. Soc. Cogn. Affect. Neurosci. 3 (2008) 177-90

38. Stam, C. J., Use of magnetoencephalography (MEG) to study functional brain networks in neurodegenerative disorders. J. Neurol. Sci. 289 (2010) 128-34

39. Zhou, Y. et al., Functional disintegration in paranoid schizophrenia using resting-state fMRI. Schizophr. Res. 97 (2007) 194-205

40. Chanraud, S., Pitel, A.-L., Pfefferbaum, A. \& Sullivan, E. V, Disruption of functional connectivity of the default-mode network in alcoholism. Cereb. Cortex 21 (2011) 2272-81

41. Orban, C. et al., Resting state synchrony in anxietyrelated circuits of abstinent alcohol-dependent patients. Am. J. Drug Alcohol Abuse 39 (2013) 43340

42. Acharya, U. R., S, V., Bhat, S., Adeli, H. \& Adeli, A., Computer-aided diagnosis of alcoholism-related EEG signals. Epilepsy Behav. 41C (2014) 257-263

43. Lopes da Silva, F., EEG and MEG: relevance to neuroscience. Neuron 80 (2013) 1112-28

44. Varela, F., Lachaux, J. P., Rodriguez, E. \& Martinerie, J., The brainweb: phase synchronization and large-scale integration. Nat. Rev. Neurosci. 2 (2001) 229-39

45. Bardin, J., Neuroscience: Making connections. Nature 483 (2012) 394-6

46. Pereda, E., Quiroga, R. Q. \& Bhattacharya, J., Nonlinear multivariate analysis of neurophysiological signals. Prog. Neurobiol. 77 (2005) 1-37

47. Mormann, F., Lehnertz, K., David, P. \& E. Elger, C., Mean phase coherence as a measure for phase synchronization and its application to the EEG of epilepsy patients. Phys. D Nonlinear Phenom. 144 (2000) 358-369

48. Klimesch, W., Doppelmayr, M., Russegger, H., Pachinger, T. \& Schwaiger, J., Induced alpha band power changes in the human EEG and attention. Neurosci. Lett. 244 (1998) 73-6

49. Klimesch, W., EEG-alpha rhythms and memory processes. Int. J. Psychophysiol. 26 (1997) 319-40

50. Klimesch, W., EEG alpha and theta oscillations reflect cognitive and memory performance: a review and analysis. Brain Res. Brain Res. Rev. 29 (1999) 169-95

51. Lee, S. et al., Disruption of orbitofronto-striatal functional connectivity underlies maladaptive persistent behaviors in alcohol-dependent patients. Psychiatry Investig. 10 (2013) 266-72

52. Rogers, B. P., Parks, M. H., Nickel, M. K., Katwal, S. B. \& Martin, P. R., Reduced fronto-cerebellar functional connectivity in chronic alcoholic patients. Alcohol. Clin. Exp. Res. 36 (2012) 294-301 
53. Park, S. Q. et al., Prefrontal cortex fails to learn from reward prediction errors in alcohol dependence. J. Neurosci. 30 (2010) 7749-53

54. Courtney, K. E., Ghahremani, D. G. \& Ray, L. a, Fronto-striatal functional connectivity during response inhibition in alcohol dependence. Addict. Biol. 18 (2013) 593-604

55. De Bruin, E. a et al., Abnormal EEG synchronisation in heavily drinking students. Clin. Neurophysiol. 115 (2004) 2048-55

56. Becoña, E. et al., La escala breve de evaluación del Síndrome de Dependencia de la Nicotina ( NDSS-S ) en fumadores españoles. Psicothema 23 (2011) 126-132

57. Taulu, S. \& Simola, J., Spatiotemporal signal space separation method for rejecting nearby interference in MEG measurements. Phys. Med. Biol. 51 (2006) 1759-68

58. Oostenveld, R., Fries, P., Maris, E. \& Schoffelen, J. M., FieldTrip: Open source software for advanced analysis of MEG, EEG, and invasive electrophysiological data. Comput. Intell. Neurosci. 2011 (2011) 156869

59. López, M. E. et al., MEG spectral analysis in subtypes of mild cognitive impairment. Age (Dordr). (2014) doi:10.1007/s11357-014-9624-5

60. Jelic, V. et al., Quantitative electroencephalography in mild cognitive impairment: longitudinal changes and possible prediction of Alzheimer's disease. Neurobiol. Aging 21 (2000) 533-540

61. Maris, E. \& Oostenveld, R., Nonparametric statistical testing of EEG- and MEG-data. $J$. Neurosci. Methods 164 (2007) 177-190

62. Cuesta, P. et al., Source Analysis of Spontaneous Magnetoencephalograpic Activity in Healthy Aging and Mild Cognitive Impairment: Influence of Apolipoprotein E Polymorphism. J. Alzheimers. Dis (2014) doi:10.3233/JAD-140633

63. Coullaut-Valera, R. et al., Drug polyconsumption is associated with increased synchronization of brain electrical-activity at rest and in a counting task. Int. J. Neural Syst. 24 (2014) 1450005

64. Rangaswamy, M. et al., Theta power in the EEG of alcoholics. Alcohol. Clin. Exp. Res. 27 (2003) 60715

65. Porjesz, B. et al., The utility of neurophysiological markers in the study of alcoholism. Clin.

Neurophysiol. 116 (2005) 993-1018

66. Saletu-Zyhlarz, G. M., Differences in Brain Function Between Relapsing and Abstaining Alcohol-Dependent Patients, Evaluated By Eeg Mapping. Alcohol Alcohol. 39 (2004) 233-240

67. Gómez, C., Pérez-Macías, J. M., Poza, J., Fernández, A. \& Hornero, R., Spectral changes in spontaneous MEG activity across the lifespan. $J$. Neural Eng. 10 (2013) 066006

68. Niedermeyer, E., The Normal EEG of the Waking Adult. Basic principles, clinical applications, and related fields, 167 (1999) at <http://www.ccs.fau.edu/ bressler/EDU/NSP/Refere nces/Niedermeyer_1999.pdf>

69. Whitford, T. J. et al., Brain maturation in adolescence: concurrent changes in neuroanatomy and neurophysiology. Hum. Brain Mapp. 28 (2007) 228-37
70. Howell, N. A. et al., Increased ventral striatal volume in college-aged binge drinkers. PLoS One 8 (2013) e 74164

71. Squeglia, L. M. et al., Binge drinking differentially affects adolescent male and female brain morphometry. Psychopharmacology (Berl). 220 (2012) 529-39

72. Doallo, S. et al., Larger mid-dorsolateral prefrontal gray matter volume in young binge drinkers revealed by voxel-based morphometry . PLoS One i (2014) 1-34

73. Schürmann, M. \& Başar, E., Functional aspects of alpha oscillations in the EEG. Int. J. Psychophysiol. 39 (2001) 151-8

74. Klimesch, W., Doppelmayr, M. \& Hanslmayr, S., Upper alpha ERD and absolute power: their meaning for memory performance. Prog. Brain Res. 159 (2006) 151-65

75. Sneider, J. T., Cohen-Gilbert, J. E., Crowley, D. J., Paul, M. D. \& Silveri, M. M., Differential effects of binge drinking on learning and memory in emerging adults. J. Addict. Res. Ther. Suppl 7 (2013)

76. Rosen, B. Q. et al., Oscillatory spatial profile of alcohol's effects on the resting state: Anatomicallyconstrained MEG. Alcohol 48 (2014) 89-97

77. Courtney, K. E. \& Polich, J., Binge drinking effects on EEG in young adult humans. Int. J. Environ. Res. Public Health 7 (2010) 2325-36

78. Chen, A. C. N., Feng, W., Zhao, H., Yin, Y. \& Wang, P., EEG default mode network in the human brain: spectral regional field powers. Neuroimage $\mathbf{4 1}$ (2008) 561-74

79. De Bruin, E. a, Stam, C. J., Bijl, S., Verbaten, M. N. \& Kenemans, J. L., Moderate-to-heavy alcohol intake is associated with differences in synchronization of brain activity during rest and mental rehearsal. Int. J. Psychophysiol. 60 (2006) 304-14

80. Del Río, D. et al., Efficiency at rest: magnetoencephalographic resting-state connectivity and individual differences in verbal working memory. Int. J. Psychophysiol. 86 (2012) 160-7

81. Klimesch, W., Sauseng, P. \& Hanslmayr, S., EEG alpha oscillations: the inhibition-timing hypothesis. Brain Res. Rev. 53 (2007) 63-88

82. Jensen, O. \& Mazaheri, A., Shaping functional architecture by oscillatory alpha activity: gating by inhibition. Front. Hum. Neurosci. 4 (2010) 186

83. Scheeringa, R., Petersson, K. M., Kleinschmidt, A., Jensen, O. \& Bastiaansen, M. C. M., EEG $\alpha$ power modulation of fMRI resting-state connectivity. Brain Connect. 2 (2012) 254-64

84. Seeley, W. W. et al., Dissociable intrinsic connectivity networks for salience processing and executive control. J. Neurosci. 27 (2007) 2349-2356

85. Petrides, M., Lateral prefrontal cortex: architectonic and functional organization. Philos. Trans. R. Soc. Lond. B. Biol. Sci. 360 (2005) 781-95

86. Buckner, R. L., Andrews-Hanna, J. R. \& Schacter, D. L., The brain's default network: anatomy, function, and relevance to disease. Ann. N. Y. Acad. Sci. 1124 (2008) 1-38

87. Spreng, R. N., Stevens, W. D., Chamberlain, J. P., Gilmore, A. W. \& Schacter, D. L., Default network activity, coupled with the frontoparietal control network, supports goal-directed cognition. Neuroimage 53 (2010) 303-17 
88. Fox, M. D. et al., The human brain is intrinsically organized into dynamic, anticorrelated functional networks. Proc. Natl. Acad. Sci. U. S. A. 102 (2005) 9673-9678

89. Jensen, O., Bonnefond, M. \& VanRullen, R., An oscillatory mechanism for prioritizing salient unattended stimuli. Trends Cogn. Sci. 16 (2012) 200-6

90. Crews, F. T. \& Boettiger, C. A., Impulsivity, frontal lobes and risk for addiction. Pharmacol. Biochem. Behav. 93 (2009) 237-47

91. Meule, A., Lutz, A. P. C., Vögele, C. \& Kübler, A., Impulsive reactions to food-cues predict subsequent food craving. Eat. Behav. 15 (2014) 99-105

92. Orr, C. et al., Altered resting-state connectivity in adolescent cannabis users. Am. J. Drug Alcohol Abuse 39 (2013) 372-81

93. Kaplan, R. F., Glueck, B. C., Hesselbrock, M. N. \& Reed, H. B., Power and coherence analysis of the EEG in hospitalized alcoholics and nonalcoholic controls. J. Stud. Alcohol 46 (1985) 122-7

94. Fingelkurts, A. a et al., Increased local and decreased remote functional connectivity at EEG alpha and beta frequency bands in opioid-dependent patients. Psychopharmacology (Berl). 188 (2006) $42-52$

95. Camchong, J., Stenger, V. A. \& Fein, G., Resting state synchrony in long-term abstinent alcoholics with versus without comorbid drug dependence. Drug Alcohol Depend. 131 (2013) 56-65

96. Campanella, S. et al., Increased cortical activity in binge drinkers during working memory task: a preliminary assessment through a functional magnetic resonance imaging study. PLoS One $\mathbf{8}$ (2013) e62260

97. Schweinsburg, A. D., McQueeny, T., Nagel, B. J., Eyler, L. T. \& Tapert, S. F., A preliminary study of functional magnetic resonance imaging response during verbal encoding among adolescent binge drinkers. Alcohol 44 (2010) 111-7

\section{ACKNOWLEDGEMENTS}

This study was supported by the project, SPI/2010/134 and SPI/2010XXX, from the Spanish Ministry of Health and Social Politics (National Plan on Drugs), and two predoctoral fellowships from the Ministry of Education (FPI, BES-2010-036469 and FPU, AP200803433).

\section{FINANCIAL DISCLOSURES}

Authors have nothing to declare. 